\title{
Introduzione allo studio della dermatologia di interesse legale
}

\author{
Valerio Cirfera ', Giancarlo Toma ${ }^{2}$, Giovanni Labrini ${ }^{3}$ \\ Specialista in Dermatologia e Venereologia. Coordinatore Nazionale AIDA-GIDeL, Responsabile Dermatologia Scienze Medico-Legali, \\ Università degli Studi di Siena [Dir.: Prof. Cosimo Loré] \\ Specialista in Medicina Legale e delle Assicurazioni, Maglie (LE) \\ 3 Già Specialista e Docente di Dermatologia e Venereologia, Parma. Fondatore dell'AIDA-GIDeL
}

\section{CAMPI DI INTERESSE}

Le scienze dermatologiche moderne, nei loro molteplici settori e campi operativi, presentano numerose tematiche di interesse medico-legale e giuridico, più di quanto comunemente si creda [1]. La realizzazione di questo numero di Pratica Medica $\&$ Aspetti Legali, interamente dedicato alla dermatologia legale, offre l'opportunità di esplicitare questa realtà ed esemplificare i rapporti intercorrenti tra la dermatologia medica, chirurgica, estetica, traumatologica, le malattie sessualmente trasmesse e il diritto. I campi di questa disciplina, anche secondo i dati delle nostre personali analisi (Tabella I), non si esauriscono nel solo ambito dell'esercizio e responsabilità professionale, ma si estendono all'accertamento e valutazione del danno biologico di natura estetica, dello stato invalidante delle dermatosi e inabilitante delle malattie professionali cutanee, agli aspetti legali delle applicazioni dell'alta tecnologia diagnostico-terapeutica e alle problematiche di sanità pubblica di natura dermo-infettivologica in ambito civile e sociale. Un ulteriore campo che negli ultimi anni ha destato notevole interesse dal punto di vista medico-legale è l'oncologia dermatologica, in ordine alla diagnosi precoce del melanoma, al suo appropriato trattamento e puntuale follow-up [2], finalizzati a salvare la vita del paziente. Altrettanto interessante è lo studio della lesività cutanea in ambito penale, in riferimento alla semeiotica nel vivente e sul cadavere, come anche il rilevamento dei caratteri inerenti l'identificazione della persona e della fenomenologia trasformativa postmortem esterna del corpo. È indubbio che, negli ultimi anni, i temi di dermatologia legale abbiano avuto un interesse sempre più crescente, destando maggiore attenzione anche da parte dei mass media, in virtù della notevole rilevanza dell'aspetto esteriore della persona nel vivere quotidiano e nei rapporti interpersonali e socio-lavorativi. La richiesta di risarcimenti per danni alla persona di ordine discromico-cicatriziale, soprattutto da infortunistica stradale, è oggi fortemente aumentata, così come l'esigenza di migliorare il proprio aspetto fisico, che ha portato ad una maggiore richiesta, rispetto al passato, di interventi estetici, valutabile fino a circa $500 \%$ in più, secondo alcune statistiche statunitensi di tutto rispetto, che hanno confrontato i dati attuali con quelli disponibili fino al 1997 [3]. L'effettuazione degli interventi estetici su larga scala ha migliorato senza dubbio la gradevolezza dell'apparire, il benessere psichico e interiore, facilitando i rapporti interpersonali; purtroppo sono aumentati anche i casi gravati da eventi avversi spiacevoli, spesso all'origine di incresciosi contenziosi per risultati prima promessi e poi non conseguiti in responsabilità contrattuale e/o danni alla persona per malpractice, in responsabilità extracontrattuale [4-7].

\section{OBIETTIVI}

Lintento della dermatologia legale è lo studio integrato e sistematico dei molteplici aspetti legali correlati alla diagnosi, terapia e gestione delle malattie e danni cutaneo-annessiali, mediante un approccio metodologico-espressivo [8] semplice e univoco, facilmente comprensibile e condivisibile nell'ambito di un contesto e di una collaborazione interdisciplinare, a cui afferiscono i contributi di figure professionali di diversa estrazione formativa e culturale, di ordine clinico, medico-legale e giuridico. Finalità similari sono state perorate, già da tempo con successo, in altre discipline, quali l'ortopedia, la ginecologia, l'ostetricia, l'ematologia, le chirurgie maggiori e altre ancora, in cui si sono costituiti gruppi di stu- 


\begin{tabular}{|lc|}
\hline Danno estetico in RCA (Responsabilità Civile Auto) & $45 \%$ \\
\hline Dermatosi professionali e invalidanti & $25 \%$ \\
\hline Responsabilità professionale & $15 \%$ \\
\hline Sanità pubblica & $10 \%$ \\
\hline Altro & $5 \%$ \\
\hline
\end{tabular}

Tabella I. Dermatologia legale: campi di applicazione più rappresentativi in ambito civile e delle assicurazioni sociali. Le percentuali derivano dalI'analisi dei dati derivanti dai casi pervenuti all'osservazione degli Autori

\begin{tabular}{|lc|}
\hline Dermatologia estetica e correttiva & $50 \%$ \\
\hline Dermatologia chirurgica e oncologica & $20 \%$ \\
\hline Dermatologia allergologica & $10 \%$ \\
\hline Dermatologia clinica e vascolare & $10 \%$ \\
\hline Terapia dermatologica off-label & $5 \%$ \\
\hline Altro & $5 \%$ \\
\hline
\end{tabular}

Tabella II. Responsabilità professionale: settori più interessati in dermatologia. Le percentuali derivano dall'analisi dei dati derivanti dai casi pervenuti all'osservazione degli Autori

dio specifici, come nel caso dell'odontoiatria e dell'oftalmologia legale, della psico-patologia e cardiologia forense, per fare solo alcuni esempi, oltre che in otorinolaringoiatria, ambito nel quale si apprezzano gli interessanti lavori effettuati [9]. La tematica fondamentale in tutti gli studi clinici di interesse legale è senza ombra di dubbio quella inerente la responsabilità sanitaria nella pratica di ogni giorno, la cui principale finalità è la conoscenza e la prevenzione dell'errore professionale prevedibile ed evitabile per la tutela, non solo della salute dei pazienti, ma anche della professione e professionalità stessa dei Sanitari, che troppo spesso vengono ingiustamente accusati in procedimenti giudiziari infondati o che tali si rivelano alla conclusione dell'inopportu- no quanto dannoso e deleterio contenzioso. Riportiamo nella Tabella II dati personali sui settori più interessati da contenzioso in dermatologia.

\section{FORMAZIONE E INFORMAZIONE TEORICO-PRATICA}

La molteplicità, l'apprezzabile incidenza e l'estremo interesse delle argomentazioni medico-legali in dermatologia hanno spinto gli scriventi a costituire [10], alcuni anni or sono, il Gruppo Italiano di Dermatologia Legale nell'ambito dell'Associazione Italiana dei Dermatologi Ambulatoriali, denominata in breve AIDA [11]. Gli obiettivi del gruppo sono quelli di approfondire e promuovere lo studio, la ricerca e la cultura nella specifica materia, attraverso l'aggiornamento di un sito web di riferimento [12], la programmazione di eventi formativi e informativi locali e nazionali, la pubblicazione di lavori ed esperienze professionali, la collaborazione alla stesura di linee guida e, infine, l'analisi e discussione della particolare casistica. In questo numero di Pratica Medica \& Aspetti Legali si riporta un'esemplificazione, sia pur non esaustiva, di quanto sopra descritto. Nello specifico, saranno pubblicate le linee guida nazionali in tema di peeling e filler, di recente stesura, sarà fatto cenno al danno alla persona di natura cicatriziale e al danno estetico in ambito penale, sarà pubblicata la prima classificazione italiana sulle forme invalidanti della psoriasi, si faranno delle riflessioni sul concetto di dermatosi occupazionali e paraprofessionali e infine si focalizzerà l'attenzione sulle implicazioni legali correlate all'effettuazione dei tatuaggi e piercing e si riporteranno alcuni casi pratici in sintesi.

\section{BIBLIOGRAFIA}

1. http://www.sidemast.org/forum.php?idTopic $=20$, forum ufficiale di dermatologia della SIDeMaST, Società Italiana di Dermatologia medica, chirurgica, estetica e delle Malattie Sessualmente Trasmesse

2. http://www.scienzemedicolegali.it/contenuto/dermatologia.html

3. ASPS Member Surgeons certified by The American Board of Plastic Surgery ${ }^{\circledR}$ as well as other physicians certified by American Board of Medical Specialties-recognized boards. ASPS 2005

4. Benci M, Cirillo PF. Gestione delle complicanze da filler, tossina botulinica e possibilità terapeutiche. Corso avanzato teorico-pratico di Dermatologia Estetica e Correttiva. Segrate, Milano

5. Labrini G. Peeling chimici. Dermatologia Ambulatoriale 2005; 13: 24-32

6. Bertana C. La gestione e l'incontro con il paziente estetico. Dermatologia Ambulatoriale 2005; 13: 74-5

7. Silvestris P, Cirfera V. Aspetti medico-legali in Dermatologia Estetica e Correttiva. Corso avanzato teorico-pratico di Dermatologia Estetica e Correttiva. Segrate, Milano.

8. http://www.dermatologialegale.it/index.php?pres=11

9. Cazzato G. Aspetti medico-legali in ORL. Associazione Otorinolaringoiatri Ospedalieri Italiani. Giofil, 2005

10. http://www.dermatologialegale.it/index.php?pres $=17$

11. http://www.aida.it

12. http://www.dermatologialegale.it 